\title{
Comparative Study of Immune-Diagnostic Tools with Polymerase Chain Reaction in Sub-Clinical Leishmaniasis Isolates
}

NMAbdalla

\begin{abstract}
:
Objective: This study aimed to identify cases of leishmaniasis in the Nuba Mountain area, which is situated in a unique geographical site located in the centre of Sudanese leishmania belt. Wide range of investigations are available for detection of leishmania cases, but still the most reliable and easy test used as screening and epidemiological tool in field studies needs to be evaluated. The most commonly used conventional diagnostic methods direct microscopy and culture have some drawbacks in diagnosing subclinical cases of leishmaniasis.
\end{abstract}

Materials and methods: In this study, comparative properties of various immune-diagnostic tools with Polymerase Chain Reaction used in sub-clinical leishmaniasis isolates were explored. The immune-diagnostic tools involved in this study include- Leishmanin Skin Test (LST), Enzyme Linked Immunosorbent Assay (ELISA) and Direct Agglutination Test (DAT). The study was conducted in the Green Valley village (Rashad Province, South Kordofan State) with a population of 332. Most of the villagers presented with sub-clinical form of leishmaniasis with minor symptoms and signs without the features found in clinical form of visceral leishmaniasis such as fever, diarrhoea, epistaxis, enlarged lymph nodes, spleen and liver.

In this study we collected demographic, clinical and epidemiological data using special questionnaire. Leishmanin skin test (LST), ELISA, DAT and PCR for parasite DNA detection were used.

Result: The final positive cases detected by PCR were 32 out of 332 belong to L. donovani species. The final positive cases detected by LST were 5 I.2\% of the total population under study, while I I out of the 37 tested samples were positive by ELISA. All of the 332 villagers showed negative readings by DAT with exception of three individuals who were positive with very high titers.

Conclusion : DNA etxtraction and amplification with primers can be a good screening tool in subclinical leishmaniasis isolates.

Keyword: Sub-clinical, Leishmaniasis, Leishmanin Skin Test, ELISA, DAT, PCR.

\section{Introduction:}

Leishmaniasis is an important parasitic disease as a major public health problem in many countries. The clinical presentation of the disease depends mainly on parasite genotype versus host immunogenetic profile. In old world transmission to human is via a bite of female sandfly mainly belonging to Phlebatomous spp. (papatasi, orientalis). L. donovani complex (donovani, infantum and chagasi) causes visceral leishmaniasis while L. tropica (arthroponotic) and L. major (zoonotic) are responsible for cutaneous leishmaniasis. ${ }^{1}$

Leishmania donovani is the main causative species for visceral leishmaniasis while Leishmania major needs an animal reservoir and it the main causative parasite for cutaneous leishmaniasis in Sudan. ${ }^{2}$ In Sudan, the leishmania belt extends from Gadarif region from east, where visceral leismania is a predominant form to Darfur region to the west, where the cutaneous form has been reported. ${ }^{3}$ The Nuba Mountain is situated in Kordofan region which lies in the mid way in the leishmania belt, this area has rarely been studied, while several studies in the field of leishmaniasis have been conducted, mainly in the eastern and southern Sudan. ${ }^{4-7}$

The leishmania antigen has been documented to yield specificity of $98 \%$ and sensitivity of $99 \%$ in serological leishmania tests. ${ }^{8}$ The optic density (O.D), spectrophotometric absorbent value of 1:100 dilution of samples serum with acute kala-azar is said to be $0.250 \pm 0.100$ and that serological test cannot predict which case will develop acute visceral leishmaniasis and which resolve without acute illness. Usually ELISA readings of specific samples decreased in subsequent years of the infection. The direct agglutination test (DAT) is used to measure the

Department of Microbiology, College of Medicine, King Khalid University, Saudi Arabia.

Correspondence: Abdalla NM, Department of Microbiology, College of Medicine, King Khalid University, Abha, P.O. 641 zip 61421, Saudi Arabia. Tel: 009662417525 Fax: 0096672247800 E-mail: uofgnazar@hotmail.com 
leishmania antibodies titer. ${ }^{9}$ The leishmanin skin test LST detects the hypersensitivity reaction (cellular immunity) towards leishmania antigens, while PCR is the new powerful diagnostic tool that depends on extraction of the nucleic acid and identifying the species-specific nucleic acid. ${ }^{10,11}$

The study explores some comparative properties of variable immune-diagnostic tools with Polymerase Chain Reaction used in sub-clinical leishmaniasis isolates.

\section{Material and Methods:}

The work site. A small village lies in Nuba Mountain, west of Sudan. The total population was around 332. The area was selected due to its unique geographical site being in the middle of Sudan leishmania belt.

Sampling: Numbering of houses (cottages) in each village and identification numbers for all villagers were performed. Three consecutive field interventions were performed in months August, October and February as they reflect the summer, winter and autumn respectively or pre-raining, raining and post-raining seasons, as these periods affect greatly the breeding of the disease vector (sandflies) so the variation of the vector population will lead to variation in transmission rate.

Demographic, epidemiological and clinical data. These data were collected using special questionnaire. Clinical examination of all villagers were conducted (by the corresponding author as he is a medical professional). Screening for the symptoms and signs related to leishmaniasis included fever, epistaxis, abdominal pain, anaemia, enlarged liver, spleen and lymph nodes.

Leishmanin skin test (LST). (donated kindly by Prof. ElHassan, Sudanese leishmaniasis research group-LRG, Khartoum, Sudan) It was done in all villagers in three consecutive occasions August (summer), February (winter), October (autumn). $0.1 \mathrm{ml}$ leishmanin was injected subcutaneously in the upper extensor part of the left arm. The ball pin technique was applied after 48-72 hours. Indurations, redness and swelling more than $5 \mathrm{~mm}$ was considered positive. The LST grades were applied to blot the test results.

ELISA. Polystryene micro-ELISA plates were coated with antigen by adding to each well $0.1 \mathrm{ml}$ of diluted leishmania antigen in $0.05 \mathrm{M}$ bicarbonate buffer with $0.02 \% \mathrm{NaN}_{3}, \mathrm{pH}$ 9.6. The plates were incubated at $4 \mathrm{C}^{\circ}$ overnight. The wells were blocked with egg white buffer (EWB), for $30 \mathrm{~min}$. at room temp. after which the antigen solution was decanted and the plates were washed three times each for $3 \mathrm{~min}$. with $0.01 \mathrm{M}$ phosphate-buffered saline and $0.05 \%$ Tween 20 (PBSTween), $\mathrm{pH} 7.2$ the plates were vigorously shaken to remove all residual fluid. Test sera were diluted in the microtiter plate wells in duplicate by adding $2 \mu \mathrm{l}$ of serum or $40 \mu \mathrm{l}$ of filter paper eluted respectively. To $0.2 \mathrm{ml}$ or $0.6 \mathrm{ml}$ of PBS-Tween so all samples were screened at a final dilution of 1:100. These plates were incubated at room temp. for $1 \mathrm{hr}$. and washed with PBS-Tween as before. To each well $0.2 \mathrm{ml}$ of conjugate 110,000 in PBS-Tween was added. The plates were incubated again for $1 \mathrm{hr}$. at room temperature after washing 3 times with PBS-Tween, $0.2 \mathrm{ml}$ of substrate solution was added to each well and incubated in dark at room temperature at the end of $30 \mathrm{~min}$. The reaction stopped by addition of $1 \mathrm{~N} \mathrm{HCL}$ or $100 \mu \mathrm{l}$ 5\%SDS (sodium dodecyl sulphate) to each well. Results were read visually and precisely by means of photometer reading the optic density (O.D) at $405 \mathrm{~nm}$.

DAT. The direct agglutination test antigen used was kindly provided by El-Harith (AMC, Department of Medical Microbiology, Laboratory of Parasitology, Amsterdam, Netherlands). The test was performed according to El-Harith protocol which involve addition of $0.8 \%$ of $0.1 \mathrm{M} 2-$ mercaptoethanol to the diluent. A titer $\geq 1: 3200$ was considered positive. Finger prick blood samples were dried on filter papers were illuted on the first day by taking one punch into $200 \mu \mathrm{l} \mathrm{NaCl} 0.9$ and incubated overnight at $4 \mathrm{C}^{\circ}$, serial dilution of each sample was prepared; $5 \mu$ blood sample in $200 \mu \mathrm{l} \mathrm{NaCl}$. $2.5 \mu \mathrm{l}$ serum in $200 \mu \mathrm{l} \mathrm{NaCl}$ (1:10 dilution), the elutes were kept at $4 \mathrm{C}^{\circ}$. On the second day, the dilution fluid $0.9 \mathrm{gm} \mathrm{NaCl}+0.2 \mathrm{gm}$ of gelatin in $100 \mathrm{ml}_{2} \mathrm{O}$ was made, then kept at $56 \mathrm{C}^{\circ}$ for $10 \mathrm{~min}$. , to dissolve the gelatin. $0.78 \mathrm{ml}$ of 2mercaptoethanol was added, the micro plates wells filled with the dilution fluid, the first well with 75 ul dilution $+50 \mathrm{ul}$ of the elution of the filter paper. The dilution to start with was $1: 200$. The other wells were filed with 50 ul of the dilution fluid. For the first screening, dilution was done with the multi-channels pipette, started in the first well, mixed and 50 $\mu \mathrm{l}$ was taken to the next well each time. $50 \mu \mathrm{l}$ was taken off at the end. In this first screen only four steps of each sample were involved; 1:200, 1:400, 1:800 and 1:1600. $50 \mu \mathrm{l}$ antigen was added to each well, the plates were taped gently on all sides then left for 18 hours at $20 \mathrm{C}^{\circ}$ (agglutination reaction goes faster at higher temperature). On the third day, the microtiter plates were read against a white background. The titer was determined by comparison of the compact, clean spot and that of the control spot. The second screening was done to samples with titers $>1600$, these samples were diluted to more higher dilution titer.

$\boldsymbol{P C R}$. A finger prick blood spotted filter papers were collected from all the villagers. All the samples were tested for leishmania parasite detection using specific donovani primers AJS3 (5'CCAGTTTTTCCCGCCCCT3') \& DB8 (5' GGGTTGGTGTAAAATAGGGC3) (Barker, Cambridge).

DNA extraction: DNA was extracted from blood spotted filter papers collected from all the villagers. SDS detergent and proteinase $\mathrm{K}$ were used, after spinning the supernants were treated with phenol/chloroform/isoamyl alcohol. DNA were precipitated in absolute ethanol and preserved in Tris-EDTA (TE). The chelex extraction method was proved not to be 
more sensitive when compared with phenol/chloroform/ isoamyl alcohol.

Parasite DNAdetection: all samples were amplified by PCR using kDNA primers (AJS3 \& DB8) and Taq polymerase enzyme was added after the first PCR cycle "hot start" $\left(95 \mathrm{C}^{\circ}\right.$ for 5min.). After the PCR cycles ended the amplified DNA was examined using horizontal agarose electrophoresis technique visualized by Ethidium bromide. DNA profile was compared with known L.d isolates (positive control). PCR reaction conditions using Leishmania primers (AJS3 \& DB8) composed of: Hot start temp. $94 \mathrm{C}^{\circ}$ for 5 mins., Denaturation temp. $94 \mathrm{C}^{\circ}$ for $1 \mathrm{~min}$., Annealing temp. $64 \mathrm{C}^{\circ}$ for $1 \mathrm{~min}$., Extension temp. $72 \mathrm{C}^{\circ}$ for 2 mins., Incubation temp. ${ }^{2} \mathrm{C}^{\circ}$ for 10 mins., Store temp. $12 C^{\circ}$ for overnight.

Contents of $24 \mathrm{ml}$ total PCR reaction mixture: $4 \mathrm{ml} \mathrm{d}$ NTPS "Nucleotides", $2.5 \mathrm{ml} 10 \mathrm{X}$ Reaction Buffer, $1.5 \mathrm{ml}$ Primer AJS3 “dilution,” $1.5 \mathrm{ml}$ Primer DB8 “dilution”, $9.5 \mathrm{ml} \mathrm{H}_{2} \mathrm{O}, 5$ $\mathrm{ml}$ Taq-polymerase "dilution". All samples were run in $1.4 \%$ agarose gel stained with Ethidium bromide at $80 \mathrm{~V}$.

\section{Results}

Total population under study was 332; 155 children age less than 15 years and adults represents the rest (48\% of the population) females were predominant (Fig. 1). Clinically both groups (children and adults) did not complain of weight loss, epistaxis and cough. Only 3\% complained of diarrhea. Fever was a complaint of $34 \%$ of children and $16 \%$ adults, while abdominal pain of $6.7 \%$ of children and $13.5 \%$ of adults. Clinical assessment of liver and spleen enlargement among the study group 14 individuals had enlarged liver while 50 individuals had enlarged spleen. Only 63 individuals (51 children, 12 adults) showed enlarged lymph nodes, localized to inguinal region.

Positive malaria blood film was identified in $29.4 \%$ of children and $25.3 \%$ of adults where all were Plasmodium falciparum and only one case was P. malariae.

The final positive LST showed $51.2 \%$ of the population under study were exposed to leishmania parasite. $20 \%$ of children group lies in the $3^{\text {rd }}$ grade (skin reaction diameter more than $8 \mathrm{~mm}$ ), $51 \%$ adult group lies in the $3^{\text {rd }}$ grade. ELISA was done to 37 clinical suspects in this study, it showed 11 positive readings (O.D. $\geq 0.09$ ) and it seems to be more sensitive the DAT. Overall $30 \%$ of cases were positive or border line, the highest reading (0.91) was detected in the one clinical suspect case (child of 6years old).

The DAT was performed to all 332 population under study and showed only 3 positive cases. These cases were 2 children and one adult all of them showed very high titer (Table 1). The negative DAT titer reading was $\leq 1$ : 3200 .

Detection of leishmanai parasite DNA in some field samples using kinetoplast DNA primers AJS3\&DB8 (donovani species specific primers). The final positive cases detected by PCR test were 32 out of 332 belong to donovani species. Some of the positive field samples are shown in (Fig. 2). The details of some clinical suspected cases proved by PCR technique plus other positive diagnostic tools are shown in (Table 2). The DAT titer for all of these cases was 1:200 which is considered negative.

Table-I

Results of variable diagnostic tools of the three DAT positive isolates.

\begin{tabular}{lccccccc}
\hline Case No. & Age & Clinical complain & Clinical & BF Malaria & ELISA & DAT & PCR \\
\hline 181 & 48yrs & Nil & Nil & + ve & Not done & 12800 & -ve \\
182 & $9 y r s$ & Nil & Nil & +ve & Not done & 25600 & -ve \\
199 & 6yrs & Nil & Nil & -ve & Not done & 12800 & -ve \\
\hline
\end{tabular}

BF: blood film

Table-II

The suspected cases proved by PCR technique. (DAT titer for all of these cases was 1:200 which is considered negatives)

\begin{tabular}{|c|c|c|c|c|c|c|c|c|}
\hline Case No. & Age & Organ enalargement & Lymph node & BF Malaria & LST cm. & ELISA & Direct microscopy & PCR \\
\hline 20 & 8yrs & $\mathrm{S}: 1 \mathrm{~cm}, \mathrm{~L}: 0 \mathrm{~cm}$. & Positive & +ve & 0 & Not done & Not done & +ve \\
\hline 23 & $7 y r s$ & $\mathrm{~S}: 2 \mathrm{~cm}, \mathrm{~L}: 0 \mathrm{~cm}$. & Positive & -ve & 0 & Not done & Not done & +ve \\
\hline $37 *$ & $5 y r s$ & $\mathrm{~S}: 8 \mathrm{~cm}, \mathrm{~L}: 4 \mathrm{~cm}$. & Positive & -ve & 0 & $0.91+$ ve & Positive & +ve \\
\hline 38 & $7 y r s$ & $\mathrm{~S}: 0 \mathrm{~cm}, \mathrm{~L}: 0 \mathrm{~cm}$. & Positive & -ve & 0 & Not done & Not done & +ve \\
\hline 41 & $5 y r s$ & $\mathrm{~S}: 4 \mathrm{~cm}, \mathrm{~L}: 2 \mathrm{~cm}$. & Positive & +ve & 0 & $0.08-$ ve & Not done & +ve \\
\hline 131 & $9 y r s$ & $\mathrm{~S}: 8 \mathrm{~cm}, \mathrm{~L}: 0 \mathrm{~cm}$. & Positive & -ve & 0 & 0.11 -ve & Not done & +ve \\
\hline 132 & $15 y r s$ & S: $0 \mathrm{~cm}, \mathrm{~L}: 0 \mathrm{~cm}$. & Positive & -ve & 10 & Not done & Not done & +ve \\
\hline 133 & $23 y r s$ & $\mathrm{~S}: 0 \mathrm{~cm}, \mathrm{~L}: 0 \mathrm{~cm}$ & Positive & -ve & 12 & $0.11-$ ve & Not done & +ve \\
\hline 240 & $9 y r s$ & $\mathrm{~S}: 6 \mathrm{~cm}, \mathrm{~L}: 4 \mathrm{~cm}$. & Positive & +ve & 0 & $0.08-$ ve & Not done & +ve \\
\hline 385 & $2 \mathrm{yrs}$ & $\mathrm{S}: 3 \mathrm{~cm}, \mathrm{~L}: 0 \mathrm{~cm}$. & Positive & -ve & 0 & $0.06-$ ve & Not done & +ve \\
\hline
\end{tabular}

ELISA: Enzyme Linked Immunosorbent Assay, DAT: Direct Agglutinataction Test, LST: Leishmanin Skin Test, PCR: Polymerase Chain Reaction. S: spleen size, L: liver size, BF: blood film.

* Case No. 37 Is considered active visceral leishmaniasis and received treatment and cured. 


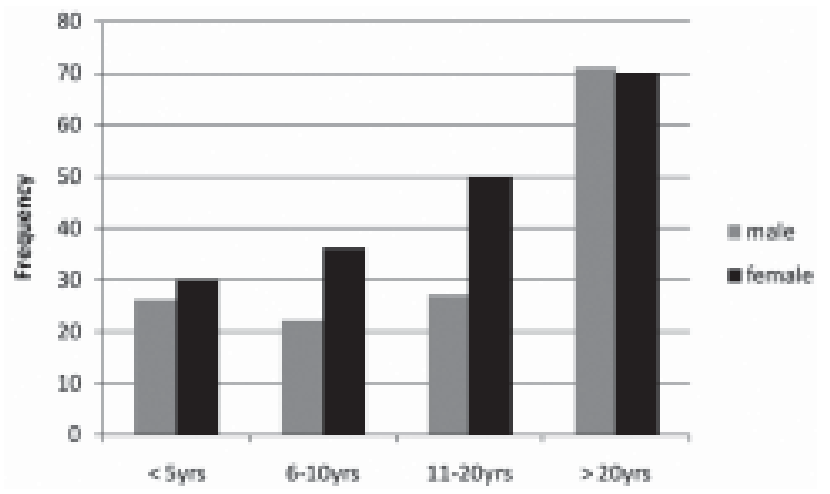

Fig.-1: Distribution of sex and age among the study group.

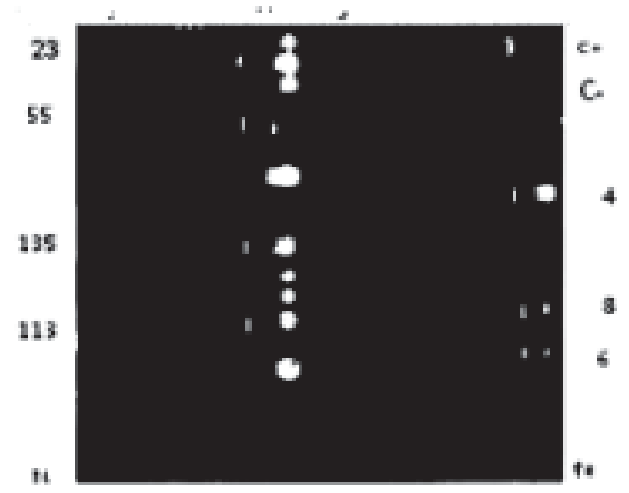

Fig.-2: AJS3\&DB8 primers used on field samples to detect leishmania DNA.

(positive samples from down upward on the left side of the gel No. 113, 135, 55, 23, M (DNA marker) and from the right side No. 6, 8, 4, C-, C+ controls.

\section{Discussion}

Leishmaniasis is a parasitic disease transmitted to human by an infected female sandfly bite of the genus Phelbotomus. ${ }^{12}$ Leishmania donovani is the main parasite species causing visceral leishmaniasis in Sudan, while Leishmania major is responsible for most infection leading to cutaneous leishmaniasis, known as zoonotic cutaneous leishmaniasis (ZCL) that confere a zoonotic reservoir. ${ }^{13}$ Leishmaniasis is a worldwide distributed and endemic in 82 countries. The annual incidence is estimated at some 600.000 new clinical cases, officially reported of 12 million cases and a population at risk about 350 million. ${ }^{14}$

The clinical pattern of the disease among the villagers understudy ( $\mathrm{n}=332$ ) showed the predominance of sub-clinical pattern. The disease clinical manifestation is governed by the host immune reaction. There are $\mathrm{T}$ cells subsets ( $\mathrm{T}$ helpers):TH1 associated with IL2, gamma IFN and mediate DTH immunity to the disease. TH2 associated with IL4, 5 and promote IgG but do not mediate DTH and most likely a florid clinical pattern of the disease. The Human HLA-pattern (locus on chromosome 6) is a major determinant of individual immune-genetic reaction in diseases and tissue typing. In the other hand the parasite genotype is also an important determinant of the microorganism virulence and disease presentation. The molecular analysis of leishmaniasis is proved to be highly sensitive and specific on clinical and sub-clinical human samples. ${ }^{15}$

In this study most of leishmania cases identified by the positive leishmanin test showed subclinical or minor clinical presentation. Epidemiological studies from endemic regions worldwide showed the predominance of cases among children in indoors infections. ${ }^{16,17}$ This is explained by the low immunity in children in comparison to adults. Kala-azar is a potentially fatal disease characterized by long term fever, spleen enlargement, immunosuppersion and weight loss. The parasites inhabit the macrophages of the spleen, liver and bone marrow in the form of aflagellated amastigotes. During the course of the disease, there is a marked depression of cellular immunity to leishmania antigens and a polyclonal B cell activation with high titer of both specific and non-specific antibodies. ${ }^{18}$ After successful treatment both $\mathrm{T}$ cell proliferation to leishmania antigens in vitro and delayed type hypersensitivity to killed leishmania promastigotes in vivo develop. ${ }^{19}$ This is shown by the low LST reaction in adults (20\% positive in $3^{\text {rd }}$ LST grade and 51\% respectively).

Leishmaniasis is mainly $\mathrm{T}$ cell-mediated disease, so selection of an efficient clinic-epidemiological tool has lead to chose the leishmanin skin test (Montonegro test; LST) which proved to be a good screening and epidemiological tool for identification of leishmania cases - clinical and sub-clinical ones. ${ }^{20}$ In comparison to other tools such as skin smear or biopsy for microscopic identification of parasites, the latter is considered as gold standard test for leishmaniasis, the majority of specimens showed negative results when the number of parasites are scarce. Molecular tools such as PCR used to be expensive. The ELISA results didn't identify the disease situation whether recent or old infection but positive results denote the exposure to the parasite antigens. Although ELISA was done to 37 clinical suspects in this study, it showed 11 positive readings (O.D. $\geq 0.09)$ and it seems to be more sensitive than DAT. Overall $30 \%$ of cases were positive or border line, the highest reading ( 0.91$)$ was detected in the one clinical suspect case (child of 6years old).

The DAT was performed to all 332 population under study and showed only 3 positive cases. These cases were 2 children and one adult all of them showed very high titer, were not correlated to any false positive factors; such as age, nutrition, malaria or any other illness (Trypansomiasis is not prevalent in the study area). 
Leismania of the donovani complex is known systematic killer pathogens, as asymptomatic infections appear to be very common. This is very well documented in the Mediterranean region, while information is more scanty in the Indian sub-continent. So far, the VL control programs in the latter region do not target the asymptomatic carriers of the infection, as there is no drug safe enough to administer in this group and as there is no validated tools to detect such carriers in the population. Comparing the performance of the direct agglutination test (DAT) and a PCR as potential markers of leishmania infection in different healthy human population from a VL endemic focus in Nepal was done to evaluate leishmania infection marker in: (1) healthy individuals without any known past history of Kala-azar and not living in the same house as known Kala-azar cases (HNK) (2) healthy household contacts of current or past VL cases (HHC) (3) healthy individuals successfully treated in the past Kala-azar episode (HPK). Very different results were encountered according to the type of healthy individuals considered. In HNK, PCR positivity was higher than DAT positivity (17.6\% and 5.6\% respectively). In HHC, $12.5 \%$ of PCR positivity and $20.8 \%$ of DAT. In HPK, DAT positivity was much higher than PCR positivity (95.7\% and 26.1\% respectively). ${ }^{21}$ This study highlights the specific character of PCR and DAT for the exploration of leishmania asymptomatic infections and suggest the usefulness of PCR for detection of very recent leishmania infection, while DAT could provide information on the log-term history of leishmania infections.

\section{Conclusion}

The traditional investigation methods used to identify leishmaniasis such as direct microscopy looking for LDBs (leishmania donovani bodies), culture and serology (ELISA, DAT and K39) have their weakness in identifying the positive cases specially if used for subclinical cases where the number of parasites will be expected to be low as well as the problems of culture contamination and cross reaction of serological tests. The DNA extraction and amplification using specific primers (Kinetoplast DNA) is a good screening tool that can be used in variable epidemiological studies. Furthermore, the species-specific causative agent can be identified and further taxonomic characteristics can be obtained.

\section{Conflict of Interest: None.}

\section{Acknowledgement}

We confer our gratitude to The Institute of Nuclear Medicine, Molecular Biology and Oncology (University of Gezira, Sudan), Institute of Endemic Diseases (University of Khartoum, Sudan) and MSF-Holland (Non governmental organization, Sudan) as they have greatly supported this field work. Our sincere thanks goes to Miss Nada Suliman and Mr. Omeran Osman for their technical support. This work was supported partially by the TDR/WHO grant (Ref.M8/181/4/A.295).

\section{References:}

1. Neave S. "Leishmania Donovani” in the Soudan. Br Med J 1904; 1(2265):1252

2. Kirk R, Sati MH. Studies in leishmaniasis in the AngloEgyptian Sudan. II. The skin and lymph glands in kala-azar. Trans R Soc Trop Med Hyg. 1940;33: 501-506

3. Zijlstra EE, El-Hassan AM,. Leishmaniasis in Sudan. Trans R Soc Trop Med Hyg. 2001; 95: (suppl. 1):S27-58

4. Abdalla NM, Ibrahim ME, El-Hassan AM, et al. Molecular Epidemiology and Clinical Study On Leishmaniasis In Nuba Mountain - Sudan. Acta Parasitologica Turcica 2002;26 (1): 23-30

5. Abdalla NM, Ibrahim ME, El-Hassan AM, et al. Seroepidemiological Study On Leishmaniasis In Nuba Mountain - Sudan. Acta Parasitologica Turcica 2000;24 (3): 228-233

6. Zijlstra EE, EL-Hassan AM, Ismail A, et al. Endemic kalaazar in Eastern Sudan; A longitudinal study on the incidence of clinical and sub-clinical infection and post kala-azar dermal leishmaniasis. Am J Trop Med Hyg 1994;51(6): 826-36

7. Siddig AM, Ghalib H, Shilington D C, Peterson EA, Khidir S. Visceral leishmaniasis in Sudan. Trop Geogr Med 1990;42: $107-112$

8. El safi SH, Peters W, El Tom B, El Kadarow A, Evans DA. Studies on the leishmaniasis in Sudan. 2- Clinical and Parasitological studies on cutaneous leishmaniasis. Trans R Soc Trop Med Hyg 1991;85: 457-64

9. El-Harith AE, Kolk AH, Kager PA, et al. Evaluation of a newly developed direct agglutination test (DAT) for serodiagnosis and seroepidemiological studies of visceral leishmaniasis comparison with IFAT and ELISA. Trans R Soc Trop Med Hyg 1987;81; 603-606

10. Ho M, Koech DK, Iha DW, Bryceson AD. Immunosuppression in Kenyan visceral leishmaniasis. Clin Exp Immunol. 1983;51: 207-14

11. Mohammad AO, Naseh MR, Mallorie H, et al. Development of species-specific PCR and PCR-restriction fragment length polymorphism assays for L. infantum/L. donovani discrimination. Exp Parasitol 2009;122:61-65

12. Killick-Kendrick R. The life-cycle of leishmania in the sandfly with special reference to the form infective to the vertebrate host. Ann Parasitol Hum Comp 1990;65 Suppl $1: 37-42$

13. El-Hassan AM, Ahmed MA, Abdul Rahim AG et al. Visceral leishmaniasis in the Sudan; clinical and hematological features. Ann Saudi Med 1990;10: 51-56 
JM Vol. 12, No. 1 Comparative Study of Immune-Diagnostic Tools with Polymerase Chain Reaction in Sub-Clinical Leishmaniasis Isolates

14. World Health Organization. Control of the leishmaniasis. Report of a WHO expert committee. 1990; WHO Technical Report Series. 793

15. Tashakori M, Kuhls K, Al-Jawabreh A, et al. Leishmania major: Genetic heterogeneity of Iranian isolates by singlestrand conformation polymorphism and sequence analysis of ribosomal DNA internal transcribed spacer. Acta Trop 2006;98:52-58

16. Jahn A, Lelmett JM, Diesfeld HJ. Seroepidemiological study on Kala-azar in Baringo District, Kenya. J Trop Med Hyg 1986;89: 91-104

17. Badaro R, Reed SG, Barral A, Orge G, Jones TC. Evaluation of the micro enzyme-linked immunosorbant assay (ELISA) for antibodies in American visceral leishmaniasis antigen selection for detection of infectinon specific responses. Am J Trop Med Hyg 1986;35: 72-78

18. Carvalho EM, Teixeira RS, Warren JD. Cell mediated immunity in American visceral leishmaniasis: reversible immunosuppression during acute infection. Infect Immune 1981;33: 498-502

19. Sacks DL, Lal SL, Shrivastava SN, Blackwell J, Neva FA. An analysis of $\mathrm{T}$ cell responsive in Indian Kala-azar. J Immunol 1987;138: 908-913

20. Liew FY. Functional heterogeneity of CD4+ T-cells in leismaniasis. Immunol Today. 1989;10: 40-45

21. Bhattarai NR, Auwera GV, RijL S, et al. DAT and PCR; a matching couple to estimate Leishmania infection. WorldLeish 2009;4; 55 